\title{
Enfermería en la atención multidisciplinaria de la hipoacusia neonatal
}

\section{Nursing in the multidisciplinary care of neonatal deafness}

\section{Autores:}

Angélica Salomé Herrera Molina ${ }^{1}$

Gabriela Elizabeth Damián Sinchiguano ${ }^{2}$

Carla Cristina Calderón Cabezas ${ }^{2}$

María Elena Robalino Rivadeneira ${ }^{3}$

'Universidad Nacional de Chimborazo, Riobamba, Ecuador

${ }^{2}$ Ministerio de Salud Pública, Riobamba, Ecuador

${ }^{3}$ Hospital Provincial General Docente Riobamba, Ecuador

Autor de correspondencia: Angélica Salomé Herrera Molina. Teléfono: 0987283190. E-mail: aherreramolina@unach.edu.ec. Dirección postal: Ave. Pedro Bedón y Puruhá. Riobamba, Ecuador.

\section{RESUMEN}

El adecuado desarrollo psicomotor del niño está determinado por diferentes procesos fisiológicos. Tomando en consideración lo antes dicho, se realizó un estudio observacional, descriptivo, transversal, mixto, con el objetivo de identificar las particularidades del accionar de enfermería en la atención multidisciplinaria dirigida a la detección precoz de la hipoacusia neonatal, en el Hospital Provincial General Docente de Riobamba, Ecuador, entre octubre de 2016 y marzo de 2017. Se trabajó con la totalidad de la población, constituida por las historias clínicas de 262 mujeres que presentaron factores de riesgos maternos de hipoacusia, las de sus recién nacidos (1177) y por las informantes clave, consideradas como tal a 34 Enfermeras que laboran en los servicios de Ginecoobstetricia y Neonatología del contexto investigativo. La información referida a las audiometrías fue tomada del registro de tamizaje neonatal realizado en el Servicio de Audiología institucional. Para determinar el nivel de información sobre los factores de riesgo que se vinculan a la enfermedad y el quehacer enfermero dirigido la detección precoz de la misma, se consideró a esas variables como cualitativas nominales dicotómicas. La incompatibilidad sanguínea, Toxoplasmosis, Rubéola, Citomegalovirus y Herpes, constituyeron los factores de riesgo mayormente observados. En el ámbito particular de Enfermería, se precisa de programas de especialización que contribuyan a la preparación de esos profesionales en el área del saber de la otorrinolaringología, lo que favorecerá el incremento de la calidad de los servicios prestados, resultantes de la atención a las necesidades de audición afectadas en ese tipo de enfermos.

Palabras clave: enfermería, hipoacusia, neonato, recién nacido. 


\section{ABSTRACT}

The adequate psychomotor development of the child is determined by different physiological processes. Taking into consideration what is mentioned above an observational, descriptive, cross-sectional, and mixed study was carried out to identify the particularities of the nursing action in multidisciplinary care in order to detect early neonatal deafness in the General Hospital of Riobamba, Ecuador, between October 2016 and March 2017. The entire population was part of the study, which was constituted by the clinical records of 262 women who presented maternal risk factors of deafness, 1177 of their newborns, and 34 nurses as key informants who work in the gynecology-obstetrics and neonatology services of the research context. The information referred to the audiometries was taken from the neonatal screening register at the Institutional Audiology Service. The variables were considered as nominal dichotomous qualitative in order to determine the level of information about the risk factors that are linked to the disease and the directed nursing task the early detection of the same. Blood incompatibility, Toxoplasmosis, Rubella, Cytomegalovirus, and Herpes were the most observed risk factors. Specialization programs are needed that contribute to the preparation of these professionals in the area of knowledge of otolaryngology in the particular field of nursing. This will favor the increase in the quality of the services provided, resulting from the attention to the needs of hearing affected in this type of patients.

Keywords: Nursing, Hearing loss, Neonate, Newborn.

\section{INTRODUCCIÓN}

La audición constituye un proceso fisiológico del ser humano que le permite a este contar con la capacidad de percibir sonidos, entenderlos y reproducirlos. Contribuye a la maduración neuropsicológica global del individuo, favoreciendo en este el desarrollo de habilidades cognitivas que posibilitan el aprendizaje. En tal sentido, el diagnóstico precoz de la hipoacusia y su intervención temprana en la primera infancia beneficia la formación intelectual y el equilibrio emocional y social de la persona. ${ }^{(1-4)}$

La sistematización de la literatura contemporánea sobre esta temática, permite observar un abanico de coincidencias al conceptualizar a la hipoacusia como la pérdida parcial o total de la capacidad de percibir o entender el sonido con uno o ambos oídos. De igual manera, diferentes autores concuerdan en reportar que en el estudio de la hipoacusia, es importante identificar si el individuo no sólo escucha el sonido; sino que también puede discriminarlo de manera que tenga sentido y traduzca una información. ${ }^{(1-8)}$

En relación con la hipoacusia neonatal, la misma es considerada un problema de salud pública de alcance mundial, no resuelto. Representa el defecto congénito y la discapacidad neurosensorial más frecuente en el recién nacido. ${ }^{(9,10)}$ Se presenta con una frecuencia que triplica al síndrome de Down, septuplica a la espina bífida y es 25 veces más común que el hipotiroidismo hereditario. ${ }^{(11,12)}$

Al respecto, la Organización Mundial de la Salud (OMS) plantea que, de cada 1000 nacidos vivos, de uno a tres neonatos padecen de problemas auditivos, lo que hace que el número de 
casos diagnosticados por hipoacusia neonatal se mantenga exponencialmente alto. De manera particular, en poblaciones de menores de cinco años de edad, el 64,4\% de las deficiencias se asocian a problemas congénitos y el $6,9 \%$ se atribuye a eventos perinatales. ${ }^{(10,13,14)}$

En el Ecuador, según datos ofrecidos por el Registro Nacional de Discapacidades, ${ }^{(15)}$ hasta diciembre de 2016 se reportaron 432209 pacientes. De ese total, la deficiencia auditiva se ubica en tercer lugar con 21,71\%, lo que representa 55665 personas con ese problema de salud, de los cuales 3070 pertenecen a la provincia de Chimborazo. ${ }^{(16,17)}$

En relación a esta última ubicación geográfica, la bibliografía revisada no ofrece suficientes evidencias de la realización de estudios locales dedicados al pesquisaje de la hipoacusia neonatal, lo que motivó la realización de una investigación multidisciplinaria e interinstitucional dirigida a ese fin; de la que en el artículo que se presenta, se ofrece una caracterización de ese problema de salud, en un contexto de estudio que presta atención de salud a población predominantemente mestiza e indígena, de procedencia urbana y rural, resultados que a futuro podrán ser tomados en consideración para el diseño de un programa de intervención temprana dirigido a pacientes con discapacidad auditiva.

\section{MATERIAL Y MÉTODOS}

Se realizó un estudio observacional, descriptivo, de corte transversal, con enfoque mixto, con el objetivo de identificar las particularidades del accionar de enfermería para contribuir a la detección precoz de la hipoacusia neonatal en el Hospital Provincial General Docente Riobamba, durante el período comprendido entre octubre 2016 y marzo 2017.

Se trabajó con la totalidad de la población de estudio, la que estuvo constituida por las historias clínicas de las 262 madres que presentaron factores de riesgos de que sus hijos padezcan hipoacusia neonatal y por los 1177 estudios de Emisiones Otoacústicas (EOA) realizados a los neonatos nacidos en el contexto de estudio durante el periodo investigativo.

Para la recolección de los datos se utilizó la técnica de revisión de documentos escritos (historias clínicas de gestantes y puérperas), para lo cual se confeccionó la respectiva guía, lo que permitió identificar las madres incluidas en el estudio, la referencia de factores de riesgos ligados a esta enfermedad, la cual afecta sus productos de la concepción.

Las particularidades referidas a la necesidad de información en el personal de Enfermería acerca de detección precoz de la hipoacusia neonatal; se identificaron mediante la aplicación de una entrevista estructurada dirigida a esos profesionales de los servicios de Ginecoobstetricia (16) y Neonatología (18) del contexto de estudio, debido a que estos forman parte del equipo multidisciplinario encargado del cuidado a la madre y el neonato en la atención a la enfermedad en estudio.

Los datos recopilados se ordenaron en una base de datos confeccionada al efecto, información que se utilizó para análisis de frecuencias absolutas y relativas. Los resultados fueron proce- 
sados bajo el cumplimiento de los principios bioéticos de la investigación científica, se respetó el anonimato de las pacientes involucradas en los documentos, así como del personal de enfermería que participó.

\section{RESULTADOS Y DISCUSIÓN}

La audición constituye uno de los elementos principales para el adecuado desarrollo psicomotor del niño, posicionamiento que se sustenta en la función de las células ciliadas externas de la cóclea, estructura que tiene la capacidad de responder a un estímulo sonoro, las que al contraerse aumentan el movimiento de la membrana basilar; amplificando, por resonancia, la señal hacia las células ciliadas internas. ${ }^{(6,7,9,14)}$

Dicha contracción genera un sonido en sentido inverso a través de la ventana oval, cadena de huesecillos y tímpano, cuya vibración puede registrarse en el conducto auditivo externo, las que son llamadas como EOA. ${ }^{(6,7,9,14)}$

En la investigación que se presenta, la determinación de la hipoacusia neonatal tomó en consideración la presencia de signos de disminución de la agudeza auditiva, determinada mediante el estudio de estas. Los resultados de este examen se consideran por la ciencia de forma dicotómica, el que mide la audición dentro de una extensión de frecuencias de sonido, para lo cual se coloca una pequeña sonda en el conducto auditivo del niño, mediante la que se emite un sonido de bajo volumen en el oído.

La cóclea responde con una emisión otoacústica, descrita como un "eco" que pasa de vuelta por el oído medio al conducto auditivo y es analizado por el aparato de evaluación. El resultado aparece en el aparato como pass (pasa) o refer (remitir) aproximadamente 30 segundos después. La información referente a ese tamizaje se muestra en las tablas 1 y en la 2 los factores de riesgos de hipoacusia neonatal presentes en madres.

Tabla 1. Resultados de los tamizajes auditivos realizados a la población de estudio

\begin{tabular}{|l|r|c|c|c|c|}
\hline \multirow{2}{*}{$\begin{array}{c}\text { Examen auditivo } \\
\text { por meses de } \\
\text { estudio } \\
\text { (N=1177) }\end{array}$} & \multicolumn{4}{|c|}{ Emisiones Otoacústicas (EO) realizadas } \\
\cline { 2 - 6 } & $\begin{array}{c}\text { No. de EO } \\
\text { realizados }\end{array}$ & $\begin{array}{c}\text { No. de } \\
\text { neonatos que } \\
\text { pasan la } \\
\text { prueba }\end{array}$ & $\%$ & $\begin{array}{c}\text { Remitir } \\
\text { No. de } \\
\text { neonatos que } \\
\text { no pasan la } \\
\text { prueba }\end{array}$ & $\%$ \\
\hline Octubre 2016 & 235 & 234 & 19,89 & 1 & 0,08 \\
\hline Noviembre 2016 & 232 & 232 & 19,71 & - & - \\
\hline Diciembre 2017 & 200 & 186 & 15,80 & 14 & 1,20 \\
\hline Enero 2017 & 236 & 226 & 19,20 & 10 & 0,85 \\
\hline Febrero 2017 & 274 & 264 & 22,42 & 10 & 0,85 \\
\hline TOTAL & $\mathbf{1 1 7 7}$ & $\mathbf{1 1 4 2}$ & $\mathbf{9 7 , 0 2}$ & $\mathbf{4 2}$ & $\mathbf{2 , 9 8}$ \\
\hline
\end{tabular}

Fuente: historias clínicas de gestantes y puérperas. Hospital Provincial General Docente de Riobamba, 2016 y 2017. 
Al analizar los resultados de las audiologías realizadas en el contexto de estudio, se observó que un bajo porcentaje de neonatos que no pasaron el examen de EOA, lo que indica que los mismos pueden presentar problemas de audición neurosensoriales dentro de la cóclea, o de audición en el oído interno.

A pesar de que el estudio de las EOA representa una herramienta objetiva para la evaluación de la audición periférica en niños pequeños, este procedimiento presenta algunas desventajas que limitan su uso, por lo que esta técnica debe combinarse con otras de tipo electrofisiológica para llegar a la definición del diagnóstico de hipoacusia neonatal. ${ }^{(3-5,7,11,13)}$ Así; lo antes dicho expresa que constituye un error afirmar que un niño tiene una pérdida auditiva, sólo porque el resultado de dicho estudio no sea favorable. Al respecto, se recomienda informarles a los padres que se deberá continuar estudiando al niño hasta definir al problema auditivo.

Sin embargo, a pesar de que el resultado refer (remitir) informado por el aparato de evaluación supone que los neonatos que no pasaron la prueba pueden presentar algún tipo de hipoacusia con el tiempo. En la búsqueda realizada en los archivos del Servicio de Audiología del contexto de estudio, ni en las historias clínicas de los recién nacidos, no se evidenció la referencia de los mismos para pruebas de Potenciales Evocados Auditivos en estado estable (PEAee).

En relación con los avances científicos, resulta de importancia la continuidad de la atención a pacientes con resultados que indican remitir en exámenes de EOA, a los que se les debe realizar PEAee, método objetivo de evaluación de la audición que expresa la respuesta cerebral ante un estímulo acústico repetitivo, la que brinda la posibilidad de determinar el umbral auditivo en un amplio espectro y de manera específica por frecuencias, lo que contribuye a identificar y cuantificar la pérdida auditiva en niños y adultos que no pueden o no quieren participar en una prueba subjetiva, como la audiometría. ${ }^{(20,21)}$

En términos de fragmentación de la continuidad de la atención de salud a los neonatos con EOA refer en el ámbito estudiado, no se pudo determinar si los 42 recién nacidos (2,98\%) pertenecen al grupo de 262 madres que presentaron factor de riesgo, debido a que el formato de valoración utilizado en el Servicio de Audiología, no cuenta con esa información.

La presencia de hipoacusia en la primera etapa del ciclo vital constituye una alteración de la que se derivan repercusiones sobre el desarrollo global del niño; por lo que su identificación precoz es fundamental. Desde esa perspectiva, resulta necesario identificar los factores de riesgo de esta enfermedad presentes en madres (tabla 2), para actuar sobre ellos y minimizar las secuelas que de los mismos se puedan derivar. 
Tabla 2. Factores de riesgo de hipoacusia neonatal presentes en madres que conformaron la muestra.

\begin{tabular}{|c|c|c|c|c|c|c|c|c|}
\hline \multirow{3}{*}{$\begin{array}{c}\text { Periodo de } \\
\text { estudio } \\
(\mathrm{N}=\mathbf{2 6 2})\end{array}$} & \multicolumn{8}{|c|}{$\mathrm{N}^{\circ}$ de madres con } \\
\hline & \multicolumn{2}{|c|}{$\begin{array}{l}\text { Factor de } \\
\text { riesgo de } \\
\text { hipoacusia } \\
\text { neonatal }\end{array}$} & \multicolumn{2}{|c|}{$\begin{array}{c}\text { Historia } \\
\text { familiar de } \\
\text { hipoacusia } \\
\text { infantil } \\
\text { permanente }\end{array}$} & \multicolumn{2}{|c|}{$\begin{array}{l}\text { Historia } \\
\text { familiar } \\
\text { Hipoacusia } \\
\text { del adulto }\end{array}$} & \multicolumn{2}{|c|}{$\begin{array}{c}\text { Consanguinidad } \\
\text { de la pareja }\end{array}$} \\
\hline & No. & $\%$ & No. & $\%$ & No. & $\%$ & No. & $\%$ \\
\hline Octubre 2016 & 23 & 8,78 & - & - & - & - & 8 & 3,05 \\
\hline Noviembre 2016 & 34 & 12,98 & 1 & 0,38 & -. & $\ldots$ & 18 & 6,87 \\
\hline Diciembre 2017 & 58 & 22,13 & - & - & -. & $\ldots$ & 33 & 12,59 \\
\hline Enero 2017 & 56 & 21,38 & -- & -- & 1 & 0,38 & 36 & 13,74 \\
\hline Febrero 2017 & 47 & 17,93 & -- & - & 1 & 0,38 & 17 & 6,49 \\
\hline Marzo & 44 & 16,80 & -- & - & 1 & 0,38 & 22 & 8,40 \\
\hline TOTAL & 262 & 100 & 1 & 0,38 & 3 & 1,14 & 134 & 51,14 \\
\hline
\end{tabular}

Fuente: historias clínicas de gestantes y puérperas. Hospital Provincial General Docente de Riobamba, 2016 y 2017.

Al analizar la correspondencia entre los antecedentes patológicos familiares tomados en consideración por la ciencia como factor de riesgo de hipoacusia neonatal, ${ }^{(22,23)}$ y los encontrados en las historias clínicas de las madres que conformaron la población de estudio, puede observarse que de forma mayoritaria la incompatibilidad sanguínea es responsable de este problema de salud en el ámbito estudiado, coincidiendo este con los identificados por otros investigadores dedicados a esta temática. ${ }^{(24-26)}$

Al respecto, autores como Mendelsberg y col., ${ }^{(24)}$ consideran que en la detección de la hipoacusia neonatal, resulta importante establecer el diagnóstico de las mutaciones en el gen de la conexina 26; resultado que favorece el manejo integral de la sordera congénita no sindrómica de forma oportuna; para lo cual se debe tomar en consideración los estudios moleculares, pues permiten conocer el perfil genotípico de este grupo de pacientes y en relación a esto orientar el asesoramiento genético y brindar cuidados específicos a la madre y al recién nacido.

En relación asociación de otros factores de riesgos con la enfermedad en estudio, no se encontró coincidencia con los reportes realizados por otros autores, los que vinculan a la hipoacusia neonatal con malformaciones craneofaciales; ${ }^{(27)}$ así como a la presencia de fiebre, infecciones y uso de medicamentos durante la gestación. ${ }^{(28-30)}$

En coincidencia con investigadores como Cofré y col., ${ }^{(28)}$ se observó la presencia de infecciones durante el embarazo en la población de estudio (tabla 3); elemento considerado como un 
factor de riesgo de fuerza mayor para causar hipoacusia en el recién nacido, debido a la relación existente entre la existencia de antecedentes patológicos personales y la enfermedad neonatal, descrita por varios autores. ${ }^{(31-34)}$

Tabla 3. Factores de riesgo de hipoacusia neonatal: antecedentes patológicos personales

\begin{tabular}{|c|c|c|c|c|c|c|c|c|c|c|}
\hline \multirow{3}{*}{$\begin{array}{l}\text { Periodo de } \\
\text { estudio } \\
(\mathrm{N}=262)\end{array}$} & \multicolumn{10}{|c|}{ Infecciones durante el embarazo } \\
\hline & \multicolumn{2}{|c|}{ Нр } & \multicolumn{2}{|c|}{$\mathbf{R b}$} & \multicolumn{2}{|c|}{ Sf } & \multicolumn{2}{|c|}{ Txp } & \multicolumn{2}{|c|}{ Cmg } \\
\hline & No & $\%$ & No & $\%$ & No & $\%$ & No & $\%$ & No & $\%$ \\
\hline Octubre 2016 & 2 & 0,76 & - & - & 1 & 0,38 & - & - & 1 & 0,38 \\
\hline Noviembre 2016 & - & - & -- & - & -- & - & 1 & 0,38 & - & - \\
\hline Diciembre 2017 & 3 & 1,14 & 3 & 1,14 & -. & - & 1 & 0,38 & 3 & 1,14 \\
\hline Enero 2017 & - & - & 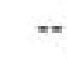 & - & - & - & - & - & - & - \\
\hline Febrero 2017 & 9 & 3,43 & 8 & 3,05 & - & - & 2 & 0,76 & 9 & 3,43 \\
\hline Marzo & 3 & 1,14 & 3 & 1,14 & -. & - & - & -- & 2 & 0,76 \\
\hline TOTAL & 17 & 6,47 & 14 & 5,33 & 1 & 0,38 & 4 & 1,52 & 15 & 5,71 \\
\hline
\end{tabular}

Fuente: historias clínicas de gestantes y puérperas. Hospital Provincial General Docente de Riobamba, 2016 y 2017.

\section{Leyenda:}

Hp: Herpes

Rb: Rubeola

\section{Sf: Sífilis}

Txp: Toxoplasmosis

\section{Cmg: Citomegalovirus}

Los antecedentes patológicos personales observados en la población investigada, muestran que la casi la cuarta parte de esta (51 pacientes para un 19,41\%) presentó algún tipo de infección durante el embarazo. Al respecto, se coincide con estudiosos del tema, ${ }^{(28-34)}$ los que plantean que las infecciones en esa etapa del ciclo de vida de la mujer que se asocian a la hipoacusia neonatal son reconocidas como el síndrome Toxoplasmosis, Rubéola, Citomegalovirus y Herpes (TORCH).

En relación con el uso de medicamentos durante el embarazo, la tabla 4 ofrece información sobre aquellos de mayor consumo en esa etapa de la vida de la mujer y en el ámbito de estudio, que con mayor frecuencia fueron identificados como aquellos incluidos entre los factores de riesgos de padecer esta enfermedad por el neonato. 
Tabla 4. Factores de riesgo de hipoacusia neonatal: antecedentes patológicos personales

\begin{tabular}{|c|c|c|c|c|c|c|c|c|c|c|c|c|c|c|}
\hline \multirow{3}{*}{$\begin{array}{l}\text { Periodo de } \\
\text { estudio } \\
(\mathrm{N}=262)\end{array}$} & \multicolumn{14}{|c|}{ Uso de medicamentos durante el embarazo } \\
\hline & \multicolumn{2}{|c|}{ Gt. } & \multicolumn{2}{|c|}{ Amk } & \multicolumn{2}{|c|}{ Mt } & \multicolumn{2}{|r|}{$\mathbf{C l}$} & \multicolumn{2}{|c|}{ Ib } & \multicolumn{2}{|c|}{ Mdp. } & \multicolumn{2}{|c|}{ Fr } \\
\hline & No & $\%$ & No & $\%$ & No & $\%$ & No & $\%$ & No & $\%$ & No & $\%$ & No & $\%$ \\
\hline 2016 & 5 & 1,91 & 1 & 0,38 & 7 & & & - & 1 & 0.38 & - & & 1 & 0,38 \\
\hline Nor & 5 & 1.91 & - & 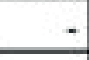 & 9 & 3.44 & 4 & 1.53 & - & - & & & & \\
\hline Dic: & 2 & 0,76 & - & . & 16 & 6,1 & 4 & 1,53 & - & - & - & & $=$ & \\
\hline Ene & 7 & 2,67 & - & -1 & 18 & 6,87 & 6 & 2,29 & - & - & - & - & 1 & 0,38 \\
\hline Feb & - & $\longrightarrow$ & - & 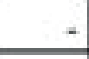 & 12 & 4,58 & 6 & 2,29 & - & - & 1 & 0.38 & - & \\
\hline Marzo & 3 & 1,15 & - & . & 15 & 5,73 & 10 & 3,817 & - & & - & & - & \\
\hline TOTAL & 22 & 8,37 & 1 & 0,38 & 77 & 29,39 & 30 & 11,45 & 1 & 0,38 & 1 & 0,38 & 2 & 0,76 \\
\hline
\end{tabular}

Fuente: historias clínicas de gestantes y puérperas. Hospital Provincial General Docente de Riobamba, 2016 y 2017

\section{Leyenda:}

Gt: Gentamicina

Amk: Amikacina

Mt: Metronidazol

\section{$\mathrm{Cl}$ : Clotrimazol}

Ib: Ibuprofeno

Mdp: Medroxiprogesterona

Fr: Furosemida

Medicamentos como el metronidazol, clotrimazol y gentamicina se reportaron como los mayormente consumidos por las madres incluidas en el estudio; factor de riesgo que, al analizarlos de forma sistémica como antecedente patológico personal, el que además incluye a las infecciones durante el embarazo y con otros de carácter familiar, como son la consanguinidad y la presencia de hipoacusia en otros miembros de la familia; revela una coincidencia de este estudio con los resultados expuestos por Fuguet y col., ${ }^{(35)}$ los que plantean que todo paciente con antecedente de exposición a medicamentos ototóxicos, sepsis o meningitis tiene alta probabilidad de adquirir algún grado de hipoacusia.

La coincidencia de resultados observados en el estudio que se presenta sobre los factores de riesgos de hipoacusia neonatal y su sincronía con lo descrito por la ciencia, reviste importancia a la indagación de las particularidades del accionar de enfermería para contribuir a la detección precoz de esta enfermedad, la cual constituye un problema de salud no resuelto en el terreno internacional, realidad presente en el Ecuador; país en el que al cierre del año 2017 se reportaron 55.020 personas con discapacidad auditiva, de los cuales 54,34\% son masculinos y $45,65 \%$ femeninos. ${ }^{(36,37)}$ 
Para alcanzar ese objetivo, la variable nivel de información del personal de enfermería sobre la hipoacusia neonatal y su detección precoz, se trató de forma cualitativa nominal dicotómica; encontrándose que, aunque el $100 \%$ de los consultados respondieron de forma favorable conocer la existencia de un protocolo al efecto y las acciones a seguir en el proceso de atención a este tipo de pacientes, reconocieron la necesidad de incrementar sus conocimientos sobre prevención, diagnóstico oportuno y el desarrollo de acciones para la atención a esta enfermedad; la que, si bien no es curable, resulta tratable y permite en el enfermo el alcance de una mejor calidad de vida.

Al respecto, la literatura científica contemporánea recoge evidencia del impacto positivo que sobre la salud individual y colectiva ofrecen los programas de intervención temprana en el tratamiento de esta problemática, los que sugieren que implementación de nuevas herramientas para mejorar la atención temprana de infantes con problemas de audición de acuerdo a las necesidades del actual escenario inclusivo. ${ }^{(38-40)}$

La sistematización de la información científica consultada sobre la necesidad de incrementar la participación del personal de enfermería en los equipos multidisciplinarios encargados de detección precoz de la hipoacusia neonatal, ${ }^{(40-43)}$ permite observar que si bien se reporta una activa participación de estos profesionales en ese quehacer asistencial; se precisa perfeccionar las mallas curriculares de pregrado, con contenidos que les permitan un mayor desempeño en ese ámbito; así como el desarrollo de estudios de postgrado al tal fin.

En relación con las intervenciones de comunicación exitosas para el cuidado a la salud en personas con deficiencia auditiva; autores como Méndez ${ }^{(42)}$ plantean que el profesional de enfermería no debe escatimar en el uso de las nuevas tecnologías dirigidas a este tipo de cuidado y experimentar en la creación de nuevos materiales enfocados en las particularidades de la atención a este tipo de pacientes desde la perspectiva de enfermería, sustentado en teorías y modelos que respalden las enseñanzas del cuidado al a la persona con necesidades de audición y comunicación afectadas, lo que favorecerá los procesos de aprendizaje a autocuidado.

\section{CONCLUSIONES}

- La incompatibilidad sanguínea constituyó el factor de riesgo mayormente observado en la población de estudio; elemento que, en consonancia con la información científica consultada, genéticamente condiciona el desarrollo de hipoacusia neonatal en hijos de padres en los que existe parentesco familiar de alta consanguinidad. De igual forma, se coincide con estudiosos del tema que plantean que las infecciones en esa etapa gestacional se asocian a esta enfermedad, encontrándose entre estas a la Toxoplasmosis, Rubéola, Citomegalovirus y Herpes como las más reportadas.

- El desarrollo de equipos multidisciplinarios encargados de la prevención precoz de la hipoacusia neonatal y la atención a ese tipo de pacientes, requiere contar con personal 
altamente calificados. En el ámbito particular de Enfermería, se precisa del desarrollo de programas de especialización que contribuyan a la preparación de estos profesionales en esa área del saber, lo que favorecerá el incremento de la calidad de los servicios prestados, resultantes de la atención a las necesidades de audición afectadas en esos enfermos.

Conflicto de intereses: los autores declaran que no existen.

Declaración de contribuciones: Herrera Molina, Calderón Cabezas, Damián Sinchiguano y Robalino Rivadeneira trabajaron en la búsqueda bibliográfica, recopilación, procesamiento e interpretación de la información; así como en la redacción de los resultados.

\section{REFERENCIAS BIBLIOGRÁFICAS}

1. Borkoski B Silvia, Falcón G Juan Carlos, Corujo S Cándido, Osorio A Ángel, Ramos M Ángel. Detección temprana de la hipoacusia con emisiones acústicas. Rev. Otorrinolaringol. Cir. Cabeza Cuello [Internet]. 2017 Jun [citado 2018 Oct 12]; 77(2): 135-143. Disponible en: h t t p s: / / s cielo.conicyt.cl/s cielo.php? script=s ci_arttext\&pi$\mathrm{d}=\mathrm{S} 0718-48162017000200003 \& \operatorname{lng}=$ es. http://dx.doi.org/10.4067/S0718-48162017000200003.

2. C Kolski. Hearing screening by community physicians in under-2 year-olds. European Annals of Otorhinolaryngology, Head and Neck Diseasesm [Internet]. 2014 [citado 2018 Oct 11]; 131: 2634. Disponible en: https:/ac.els-cdn.com/S187972961400060X/1-s2.0-S187972961400060X-main.pdf?_tid=8f953dda-6a0f-490d-9ac 1-6 4695 ceda38f\&acdnat $=1544659636$ cb80ff137480a98fe5e3dd79bb562754.

3. Campos Ana Carolina Moreno de, Shirane Henrique Yassuhiro, Takemoto Paulo Vitor Atsushi, Lourenço Edmir Américo. Triagem auditiva neonatal universal: conhecimento dos pediatras e neonatologistas em Jundiaí, São Paulo, Brasil. Braz. j. otorhinolaryngol. [Internet]. 2014 Oct [citado 2018 Ene 17]; 80(5): 379-385. Disponible en: http://www.scielo.br/scielo.php?script=sci_arttext\&pid=S1808-86942014000500379\&lng=en. $\quad \mathrm{http}: / / \mathrm{dx} .-$ doi.org/10.1016/j.bjorl.2014.07.006.

4. Suárez Alejo, Suárez Hamlet, Rosales Beatriz. Hipoacusia en niños. Arch. Pediatr. Urug. [Internet]. 2008 Dic [citado 2017 Dic 22]; 79(4): 315-319. Disponible en: h t t p : / / w w w.s cielo.edu.uy/scielo.php? s cript=sci_arttext\&pi$\mathrm{d}=\mathrm{S} 1688-12492008000400008 \& \operatorname{lng}=\mathrm{es}$.

5. Organización Mundial de la Salud. OMS | Sordera y pérdida de la audición. WHO [Internet]. 2017 [citado 2017 Dic 16]; Disponible en: http://www.who.int/mediacentre/factsheets/fs $300 /$ es.

6. Delgado J. y otros. Detección precoz de la hipoacusia infantil. Revista pediatría de atención primaria. [Internet]. 2011 [citado 2017 Dic 16]; (13)50: 279-297 p. Disponible en: http://scielo.isciii.es/pdf/pap/v13n50/12_grupo_previfand.pdf. 
7. Pozo M, Almenar A, Tapia MC, Moro M. Detección de la hipoacusia en el neonato. 2008; Protocolos Diagnóstico Terapéuticos de la AEP: Neonatología [Internet] 2016. [citado 2017 Dic 16] p. 3-36 Disponible en: https://www.aeped.es/sites/default/files/documentos/3_2.pdf.

8. Coello F, Cuevas H, Andrade E. El tamizaje auditivo neonatal en Ecuador, un compromiso ineludible. Imbiomed [Internet] 2016. [citado 2017 Dic 16] 41(1). p. 177-184. Disponible en: http://www.imbiomed.com.mx/1/1/articulos.php?method=showDetai1\&id_articulo=109413\&id_seccion=5827\&id_ejemplar=10651\&id_revista=203.

9. Milanes Pérez RI, Silva Lemus HC, Angulo Serrano B. Importancia y nuevos conceptos acerca del tamizaje auditivo neonatal. Rev Cienc Bioméd. 2013;4(1):116-24.

10. World Health Organization. Millions of people in the world have hearing loss that can be treated or prevented. Geneva: WHO; 2013.

11. Rojas Godoy AL, Gómez Gómez O, Rivas Muñoz FA. Cumplimiento de la normatividad vigente para la detección temprana de la hipoacusia neonatal. Rev Salud Pública. 2014;16(3):462-72.

12. Krauss M. K, Heider C. C, Nazar M. G, Ribalta L. G, Sierra G. M. Programa de screening auditivo neonatal universal. Experiencia de más de 10 años. Rev Otorrinolaringol Cir Cabeza Cuello [internet]. 2013 ago. [citado 23 ene. 2016];73(2): [aprox. 8 p.]. Disponible en:

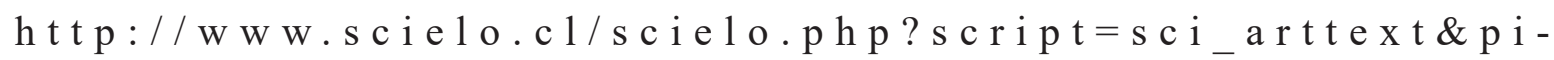
$\mathrm{d}=\mathrm{S} 0718-48162013000200003 \& \operatorname{lng}=$ es.

13. Núñez Batalla F, Trinidad Ramos G, Sequí Canet JM, Alzina de Aguilar V, Jáudenes Casaubón C. Indicadores de riesgo de hipoacusia neurosensorial infantil. Acta Otorrinolaringol Esp. 2012;63(5):382-90.

14. Blancas R, Cristina HM. Hipoacusia y factores de alarma en neonatos de alto riesgo evaluados mediante potenciales evocados auditivos. Rev Mex Neuroci. 2014 mayo-jun; 15(3): 152-6.

15. Consejo Nacional para la Igualdad de Discapacidades Ecuador CONADIS. - [Internet]. 2016 [citado 2018 Ene 6]. Disponible en: http://www.consejodiscapacidades.gob.ec/biblioteca.

16. Ministerio de Salud Pública. Registro Nacional de Discapacidades, Quito; 2016 [citado 2018 Ene 14]. p 1-19. Disponible en: https://www.consejodiscapacidades.gob.ec/wp-content/uploads/downloads/2015/09/estadistica_conadis.pdf.

17. Ruiz de la Cuesta F. Estudio del cribado neonatal y diagnóstico de la hipoacusia. Detección precoz de la hipoacusia infantil: valoración del programa de cribado y estudio de los casos diagnosticados. BOE [Internet]. 2015 [citado 2018 Ene 5];1. Disponible en: https://dialnet.unirioja.es/servlet/dctes?codigo $=64483$.

18. Navas E, Amarilis M, Hurtado V. Ni más Ni menos: Medicamentos, informándome te cuido Comunicación Organizacional y Relaciones Públicas [Internet]. Universidad San Fran- 
cisco de Quito; 2016 [citado 2017 Dic 16]. p 23-25 Disponible en: https://docplay e r.e s / $26670515-$ U n i v e r s i d a d - s a n - f r a n c i s co-de-quito-usfq-ni-mas-ni-menos-medicamentos-informandome-te-cuido.html.

19. Clavijo M, Ortiz P.: Percepciones y decisiones de los padres de los niños sordos y/o hipoacúsicos sobre la atención medica, Febrero - Julio 2016 [Internet]. Universidad de Cuenca; 2016. p. 10-15 [citado 2018 Ene 6]. Disponible en: http://dspace.ucuenca.edu.ec/bitstream/123456789/25916/1/PROYECTO DE INVESTIGACIÓN.pdf.

20. Fernández E Pamela, Marincovich S Lisette, Olivares R Virginia, Paredes S Romina, Godoy B Cristián. Aplicación de potenciales evocados de estado estable como examen auditivo en una población de jóvenes con diferentes niveles de audición. Rev. Otorrinolaringol. Cir. Cabeza Cuello [Internet]. 2009 Dic [citado 2018 Ene 17]; 69(3): 233-242. Disponible en: https://scielo.conicyt.cl/scielo.php? script=s ci_arttext\&pi$\mathrm{d}=$ S0718-48162009000300005\&lng=es.http://dx.doi.org/10.4067/S0718-48162009000300005.

21. Bidón Gómez U. Estudio comparativo coste-eficiencia de potenciales evocados auditivos automáticos frente a otoemisiones acústicas en el screening neonatal. [Tesis doctoral]. España: Universidad de Sevilla; 2017.

22. Castellanos-Coutiño, MA, Santamaría-Muñoz, R, Escobar- Carrillo, ME. Hipoacusia mediante emisiones otoacústicas en el recién nacido de la UCIN. Salud en Tabasco [Internet]. 2012 [citado 2018 Ene 11]; 18(2): 45-49. Disponible en: https://www.redalyc.org/articulo.oa? $\mathrm{id}=48724405002$.

23. Huanca Payehuanca. Emisiones otoacústicas para evaluación auditiva en el periodo neonatal y pre escolar. Paediatrica 2004: 6(1) 42-47.

24. Mendelsberg-Fishbein Paola, Márquez-Ávila Candy Sue, García-Delgado Constanza, Sánchez-Boiso Adriana, Rodríguez-Espino Benjamín Antonio, Vázquez-Martínez Edgar Ricardo et al. Importancia del diagnóstico de mutaciones en el gen de la conexina 26 en el manejo integral de la sordera congénita no sindrómica. Bol. Med. Hosp. Infant. Mex. [Internet]. 2013 Abr [citado 2018 Ene 19]; 70(2): 89-97. Disponible en: http://www.scielo.org.mx/scielo.php?script=sci_arttext\&pid=S1665-11462013000200004\&lng=es.

25. Martínez-Saucedo M, González-Huerta LM, Berruecos P, Rivera-Vega MR, Cuevas-Covarrubias SA. Análisis de la deleción del gen GJB6 en pacientes heterocigotos para el gen GJB2 con hipoacusia neurosensorial no sindrómica en una muestra de población mexicana. XXXVII Congreso Nacional de Genética Humana, Guadalajara, Jalisco. Noviembre, 2012.

26. Arenas-Sordo M, Hernández-Zamora E, Gutiérrez-Tinajero D, Murphy-Ruiz P, Leyva-Juárez X, Huesca-Hernández F, et al. Mutaciones de los genes GJB2 y GJB6 en pacientes con hipoacusia bilateral prelinguística del INR. Memorias del III Congreso Internacional de Investigación en Rehabilitación, Ciudad de México [Internet]; 2012 Nov. Disponible en: http://www.inr.gob.mx/Descargas/ciir/memorias3erCongreso.pdf. 
27. Velez-van-Meerbeke Alberto, Castelblanco Coy Lizeth. Craneosinostosis y deformidades posicionales del cráneo: revisión crítica acerca del manejo. Acta Neurol Colomb [Internet]. 2018 Sep [citado 2018 Sep 27];34(3): 204-214. Disponible en: http://www.scielo.org.co/scielo.php?script=sci_arttext\&pid=S0120-87482018000300204\&lng=en. http://dx.doi.org/10.22379/24224022214.

28. Cofré F, Delpiano L, Labraña Y, Reyes A, Sandoval A y Izquierdo G. Síndrome de TORCH: enfoque racional del diagnóstico y tratamiento pre y post natal. Recomendaciones del Comité Consultivo de Infecciones Neonatales Sociedad Chilena de Infectología, 2016. Rev Chilena Infectol [Internet]. 2016 [citado 2018 Ene 25]; 33 (2): 191-216. Disponible en: https://scielo.conicyt.cl/pdf/rci/v33n2/art10.pdf.

29. Vera Carrasco Oscar. Uso de fármacos en el embarazo. Rev. Méd. La Paz [Internet]. 2015 [citado 2018 Ago 12]; 21(2): 60-76. Disponible en: http://www.scielo.org.bo/scielo.php?script=sci_arttext\&pid=S1726-89582015000200010\&lng=es.

30. Flórez Jesús. Farmacología Humana. 6a. Edición. Barcelona España. Elsevier España 2014.

31. Ross S A, Ahmed A, Palmer A L, Michaels M G, Sánchez P J, Stewart A, et al. National Institute on Deafness and Other Communication Disorders CHIMES Study. Urine collection method for the diagnosis of congenital cytomegalovirus infection. Pediatr Infect Dis J. 2015; 34: 903-5.

32. Manicklal S, Emery V C, Lazzarotto T, Boppana S B, Gupta R K. The "silent" global burden of congenital cytomegalovirus. Clin Microbiol Rev 2013; 26: 86-102.

33. Griffiths P, Baraniak I, Reeves M. The pathogenesis of human cytomegalovirus. J Pathol 2015; 235: 288-97.

34. Kimberlin D W, Jester P M, Sánchez P J, Ahmed A, Arav-Boger R, Michaels M G, et al. Valganciclovir for symptomatic congenital cytomegalovirus disease. N Engl J Med 2015; 372: 933-43.

35. Fuguet, MV, Herize, M, Alvarado, J, Braz, ML, Zabala, M. Hipoacusia en recién nacidos con factores de riesgo perinatal. Primer Premio. LII Congreso Nacional de Pediatría 2006. Archivos Venezolanos de Puericultura y Pediatría [Internet]. 2006 [citado 2018 Ago 19]; 69(4): 137-141. Disponible en: https://www.redalyc.org/articulo.oa?id=367935535002.

36. Diario El Telégrafo [en línea]. Ecuador. La discapacidad auditiva afecta a 360 millones de personas en el mundo. Disponible en: https:/www.eltelegrafo.com.ec/noticias/socie$\mathrm{dad} / 6 /$ la-discapacidad-auditiva-afecta-a-360-millones-de-personas-en-el-mundo.

37. Ribalta L Gloria, Goycoolea V Marcos, Cardemil M Felipe, Cohen V Mauricio, Ried G Ernesto, Labatut P Tomas et al. Resultados del programa de implantes cocleares de Clínica Las Condes a 20 años de su inicio: Serie clínica 1994-2015. Rev. Otorrinolaringol. Cir. Cabeza Cuello [Internet]. 2018 Sep [citado 2018 Sep 19]; 78(3): 275-280. Disponible en: https://scielo.conicyt.cl/scielo.php? script=s ci_arttext\&pi$\mathrm{d}=\mathrm{S} 0718-48162018000300275 \& \operatorname{lng}=$ es. http://dx.doi.org/10.4067/s0717-75262018000300275. 
38. Mosnier I, Bebear J-P, Marx M, Fraysse B, Truy E, Lina-Granade G, et al. Improvement of cognitive function after cochlear implantation in elderly patients. JAMA Otolaryngol- Head Neck Surg [Internet]. 2015 [citado 2018 Ago 01]; 141: 442-50. Disponible en: https://www.ncbi.nlm.nih.gov/pubmed/25763680.

39. Suárez Alejo, Suárez Hamlet, Rosales Beatriz. Hipoacusia en niños. Arch Pediatr Urug [Internet]. 2008 [citado 2018 Dic 05]; 79(4): 315-319. Disponible en: http://www.scielo.edu.uy/scielo.php?script=sci_arttext\&pid=S1688-12492008000400008\&lng=es.

40. Sánchez Casado, JI, Benítez Merino, JM. Intervención temprana en discapacidad auditiva: diseño conceptual de "Buenas Prácticas". International Journal of Developmental and Educational Psychology [Internet]. 2013 [citado 2018 Jun 07];1(1):661-672. Disponible en: https://www.redalyc.org/articulo.oa? id=349852058056.

41. Gomes V., Correa Soares M., Manfrin Muniz R., De Sosa Silva J.R. Vivencia del enfermero al cuidar sordos y/o portadores de deficiencia auditiva. Enferm. glob. [Internet]. 2009 Oct [citado 2018 Jul 02]; (17). Disponible en: http://scielo.isciii.es/scielo.php?script=sci_arttext\&pid=S1695-61412009000300007\&lng=es.

42. Méndez-Blanco MC; Ortiz-López E; Venegas-Vindas A. Estrategias de comunicación para el personal de Enfermería que atiende personas sordas. Rev. Enferm Actual Costa Rica [Internet]. 2011 [citado 2018 Mar 14]; 21(1). Disponible en: http://bit.ly/XSwLeQ.

43. Yanchapanta Bastidas RE. La comunicación del personal de enfermería con el paciente con capacidad sensorial especial y su influencia en el cuidado del usuario, en el hospital provincial general docente Riobamba, durante el periodo de diciembre 2012-mayo 2013 [Internet]. Riobamba: Universidad Nacional de Chimborazo; 2013. Disponible en: dspace.unach.edu.ec/handle/51000/278.

Recibido: 6 de diciembre de 2018

Aceptado: 23 de diciembre de 2018 